Supporting Information

\title{
Membrane-Decorated Exosomes for Combination Drug Delivery and Improved Glioma Therapy
}

\author{
Shifu Liang, ${ }^{a}$ Huiying $X u,{ }^{b *}$ and Bang-Ce Y $e^{a, b *}$ \\ ${ }^{a}$ Institute of Engineering Biology and Health, Collaborative Innovation Center of \\ Yangtze River Delta Region Green Pharmaceuticals, College of Pharmaceutical Sciences, \\ Zhejiang University of Technology, Hangzhou 310014, China \\ ${ }^{b}$ Lab of Biosystem and Microanalysis, State Key Laboratory of Bioreactor Engineering, \\ East China University of Science and Technology, Shanghai 200237, China
}


Table S1. Encapsulation efficiency of TMZ and BG

\begin{tabular}{ccc}
\hline Drug & Method & Encapsulation efficiency (\%) \\
\hline Temozolomide & Incubation & 12.2 \\
Temozolomide & Freeze-thaw & 6.7 \\
Temozolomide & Ultrasonic & 15.8 \\
O6-Benzyl guanine & Incubation & 9.3 \\
O6-Benzyl guanine & Freeze-thaw & 6.8 \\
O6-Benzyl guanine & Ultrasonic & 15.8 \\
\hline
\end{tabular}




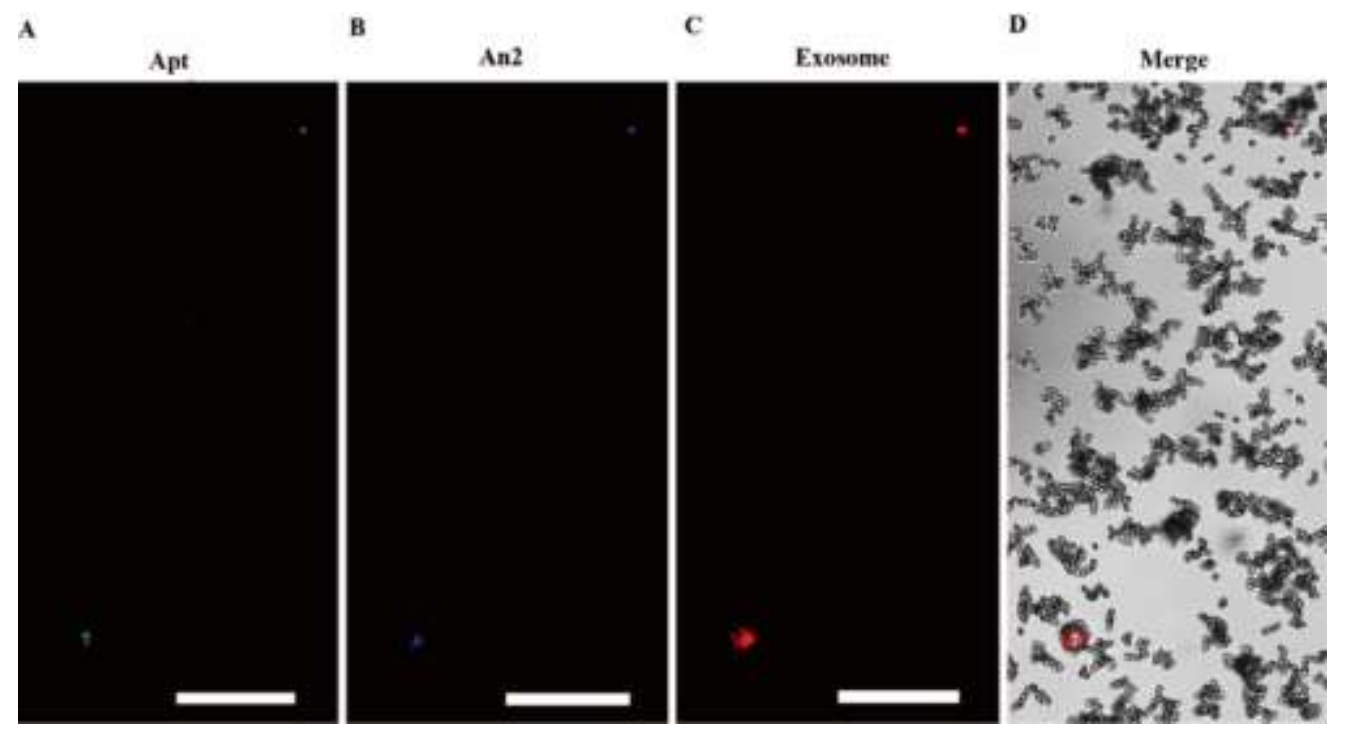

Figure S1. Confocal microscopy images of EXO (DiD)-An2(Rhodamine B)-Apt (FAM). The red spots refer to the DiD-labeled exosome excited at $630 \mathrm{~nm}$, whereas the aptamers are visualized in green at $488 \mathrm{~nm}$, the An2 links with Rhodamine B which is excited at 561 nm. Scale bar: $10 \mu \mathrm{m}$. 

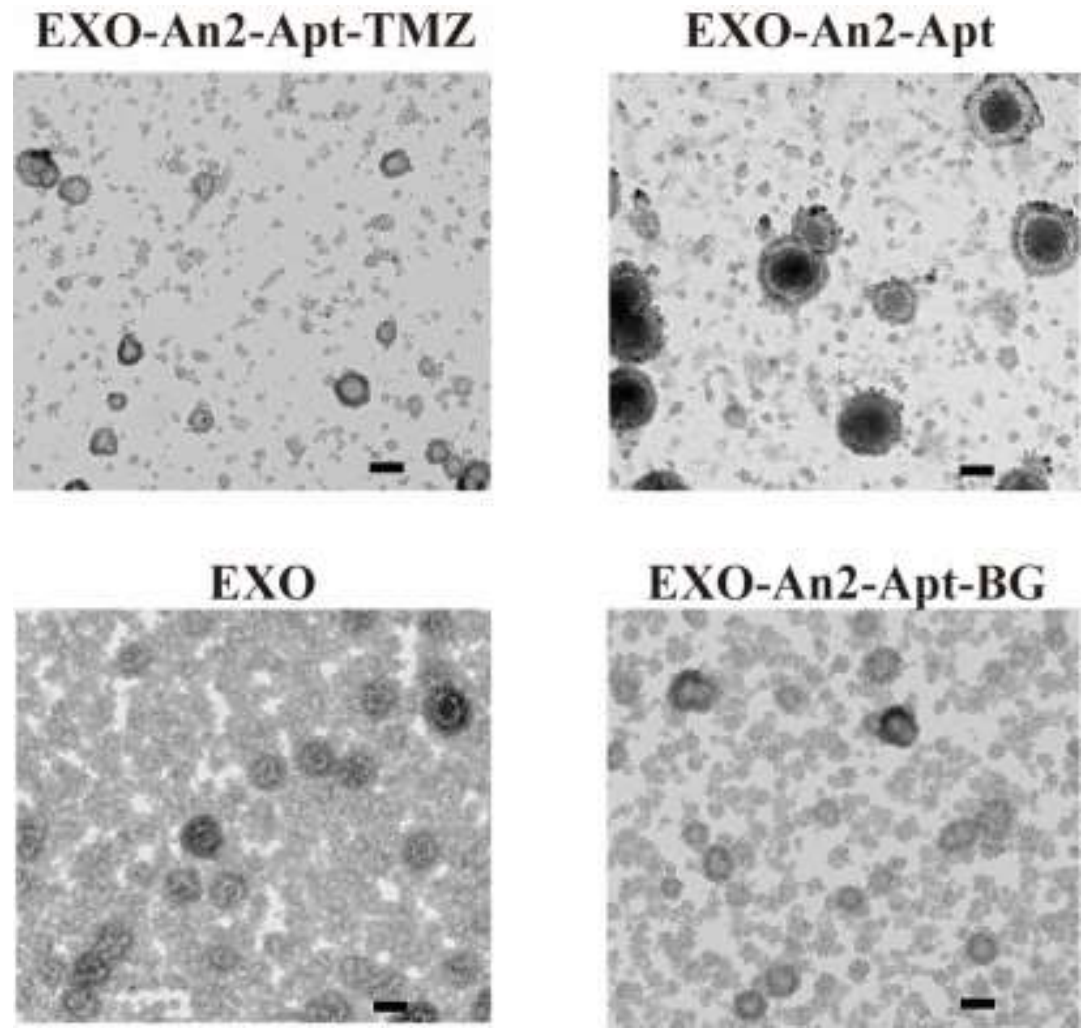

Figure S2. TEM of the engineered exosome. Scale bar: $100 \mathrm{~nm}$. 


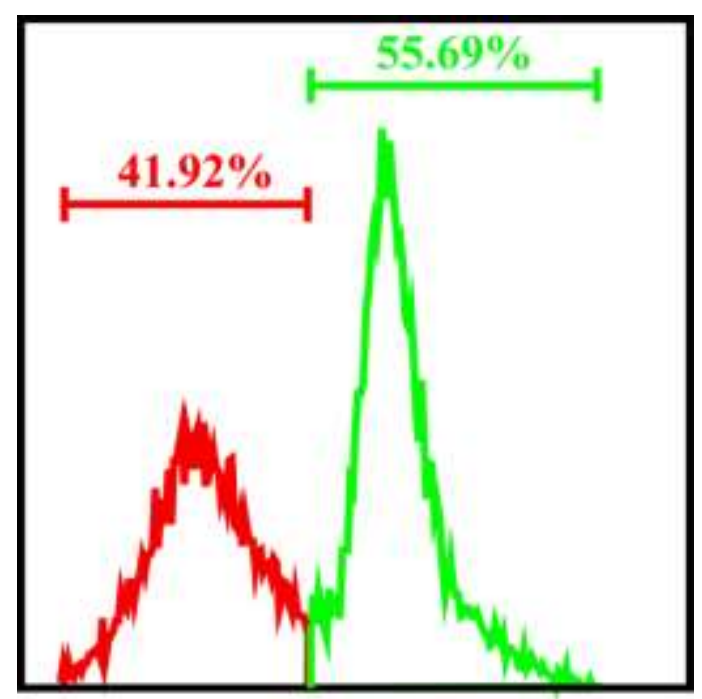

Figure S3. Flow cytometry analysis for the characterization of GSCs. 


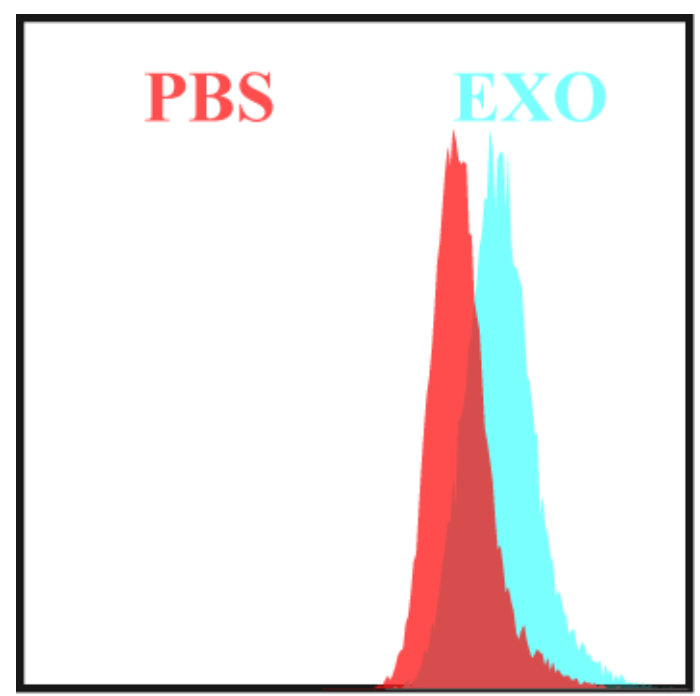

Figure S4. (A) Flow cytometric analysis of EXO-An2-Apt uptake by LO2 after $4 \mathrm{~h}$ incubation. (B) Flow cytometric analysis of LO2 without EXO-An2-Apt incubation 
A

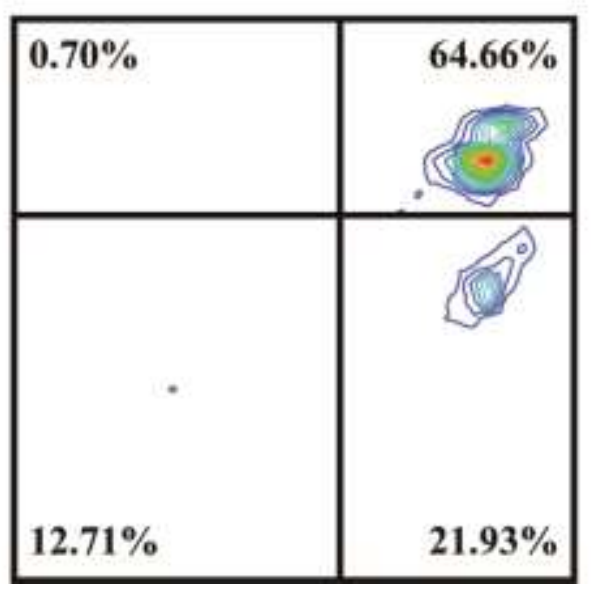

B

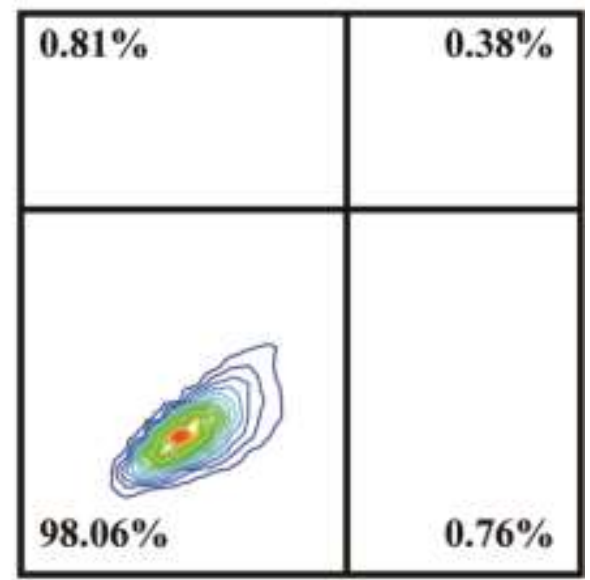

Figure S5. Flow cytometric analysis of GSCs apoptosis after incubation with $100 \mu \mathrm{M}$ EXO-An2-Apt-TMZ and $20 \mu \mathrm{M}$ EXO-An2-Apt-BG (A), and PBS (B) using Annexin VFITC/PI staining. 
A

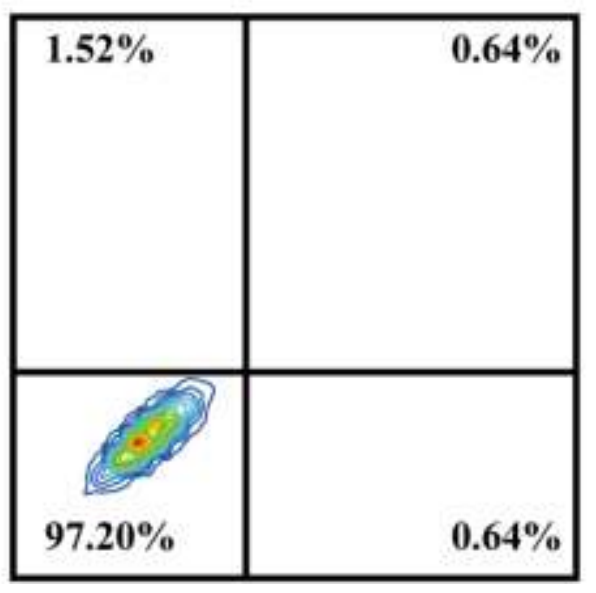

B

\begin{tabular}{|c|r|}
\hline $1.07 \%$ & $0.75 \%$ \\
& \\
& \\
\hline & \\
\hline $96.77 \%$ & $1.40 \%$ \\
\hline
\end{tabular}

Figure S6. Flow cytometric analysis of GSCs apoptosis after incubation with $100 \mu \mathrm{M}$ free TMZ and $20 \mu \mathrm{M}$ free BG (A), and PBS (B) using Annexin V-FITC/PI staining. 


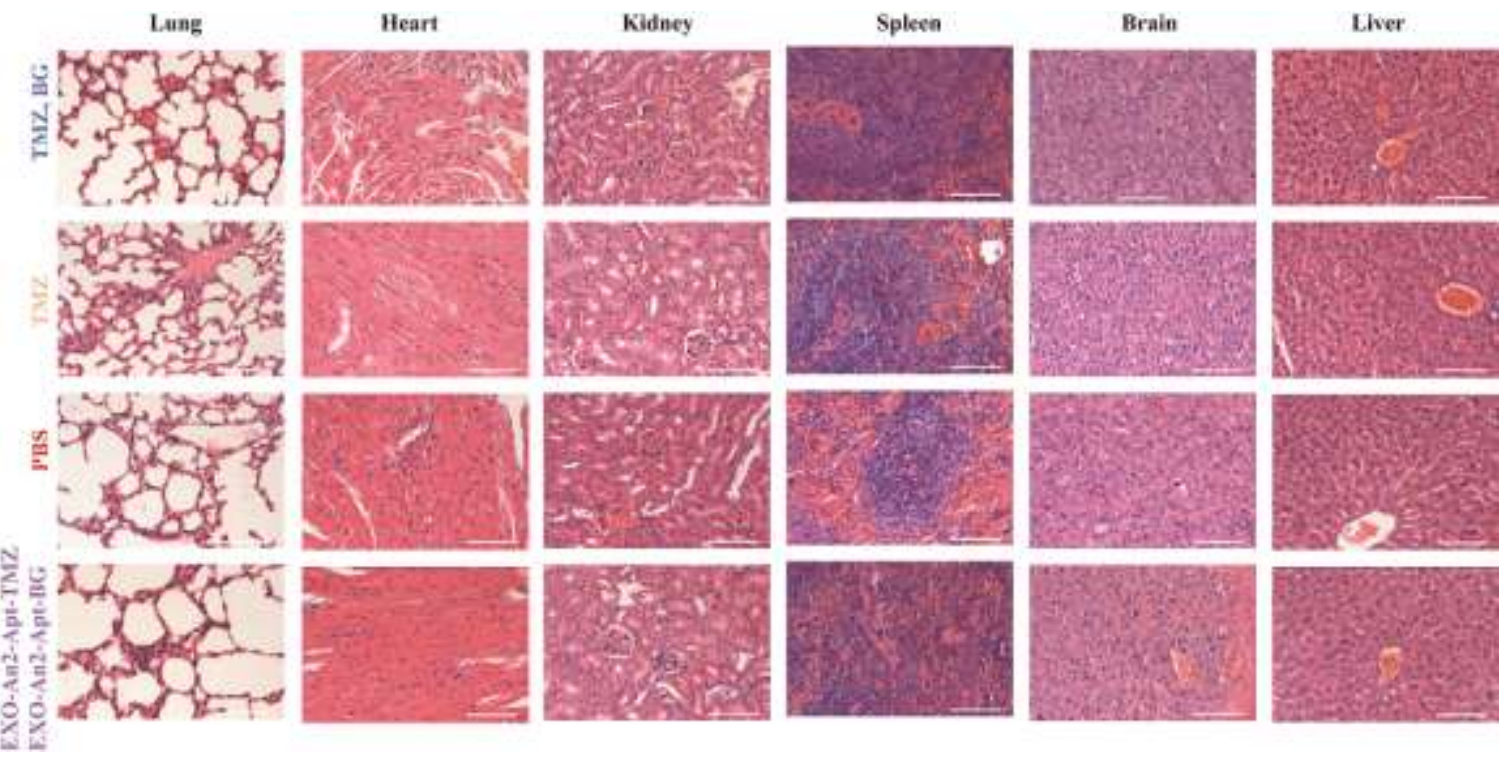

Figure S7. Histopathological examination of major organs collected after treatment on the mice bearing U87MG. 